TITLE:

\title{
Numerical study of chain conformation on shear banding using diffusive Rolie-Poly model
}

\author{
AUTHOR(S): \\ Chung, Changkwon; Uneyama, Takashi; \\ Masubuchi, Yuichi; Watanabe, Hiroshi
}

\section{CITATION:}

Chung, Changkwon ...[et al]. Numerical study of chain conformation on shear banding using diffusive Rolie-Poly model. Rheologica Acta 2011, 50(9-10): 753-766

\section{ISSUE DATE:}

2011-10

URL:

http://hdl.handle.net/2433/151858

\section{RIGHT:}

The final publication is available at www.springerlink.com; This is not the published version. Please cite only the published version.; この論文 は出版社版でありません。引用の際には出版社版をご確認ご利用くだ さい。 


\title{
Numerical study of chain conformation on shear banding using diffusive Rolie-Poly model
}

\author{
Changkwon Chung ${ }^{1}$, Takashi Uneyama ${ }^{1,2}$, Yuichi Masubuchi ${ }^{1,2}$, and Hiroshi Watanabe ${ }^{1, a)}$ \\ ${ }^{1}$ Institute for Chemical Research, Kyoto University, \\ Gokasho, Uji, 611-0011, Japan \\ ${ }^{2}$ CREST, Japan Science and Technology Agency, \\ Kawaguchi 332-0012, Japan
}

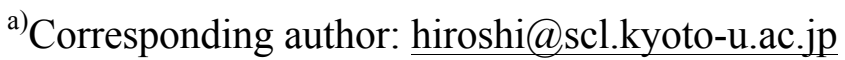

Tel: +81-774-38-8322

Fax: +81-774-38-3139

Last updated at 2011.03.14.

Manuscript to be submitted to Rheol. Acta 


\begin{abstract}
Shear-banding phenomenon in the entangled polymer systems was investigated in a planar Couette cell with the diffusive Rolie-Poly (ROuse LInear Entangled POLYmers) model, a single-mode constitutive model derived from a tube-based molecular theory. The steady state shear stress $\sigma_{\mathrm{s}}$ was constant in the shear gradient direction while the local shear rate changed abruptly, i.e., split into the bands. We focused on the molecular conformation (also calculated from the Rolie-Poly model) around the band boundary. A band was found also for the conformation but its boundary was much broader than that for the shear rate. Correspondingly, the first normal stress difference $\left(N_{1}\right)$ gradually changed in this diffuse boundary of the conformational bands. (This change of $N_{1}$ was compensated by a change of the local pressure.) For both shear rate and conformation, the boundary widths were quite insensitive to the macroscopic shear rate but changed with various parameters such as the diffusion constant and the relaxation times (the reptation time and the Rouse time). The broadness of the conformational banding, associated by the gradual change of $N_{1}$, was attributed to competition between the molecular diffusion (in the shear gradient direction) and the conformational relaxation under a constraint of constant $\sigma_{\mathrm{s}}$.
\end{abstract}

Keywords: shear banding, Rolie-Poly model, velocity band, molecular orientational band, molecular diffusion and relaxation 


\section{INTRODUCTION}

Under simple shear flow, band structures of local flow rate are often observed in several complex fluids such as wormlike micelles (Lerouge et al. 2004; Miller and Rothstein 2007; Salmon et al. 2003), polymer solutions and blends (Fielding and Olmsted 2003a, b; Furukawa and Onuki 2005; Jupp and Yuan 2004; Takenaka et al. 2006). In these fluids, the concentration and molecular orientation serve as "coupled order parameters". Focusing on these parameters, various attempts have been made to understand the shear banding phenomenon. For example, the stability analysis (Furukawa and Onuki 2005) suggested that homogeneous polymer solutions (not far from the phase separation point at equilibrium) can separate into phases having different concentrations and flow rates thereby forming the band structure. For wormlike micellar systems, the shear banding can be similarly related to the flow-induced phase separation, or, to the flow-induced nematic-isotropic (NI) transition (Berret et al. 1994; Cappelaere et al. 1997; Fischer and Callaghan 2001; Liberatore et al. 2006; Schmitt et al. 1995). (Note however that the shear banding may occur even in the absence of the flow-induced phase separation/ordering, as argued for entangled polymer solutions (Tapadia and Wang 2004, 2003).)

From a phenomenological point of view, dynamics of complex fluids is described by a constitutive relationship(s) between the strain rate tensor and the stress tensor. Thus, apart from the molecular view, we may understand some aspect of the shear banding through analysis of this relationship, for example, through the linear stability analysis (Yerushalmi et al. 1970). In this analysis, a non-monotonic constitutive relationship giving a negative slope in the steady stress $v s$ shear rate plot is believed to be the origin of the shear banding because the negative slope leads to a mechanical instability thereby forcing the fluid to split into stable bands (Lu et al. 2000; Yerushalmi et al. 1970). For the wormlike micelles, this scenario has been supported by the analysis of the diffusive Johnson-Segalman (JS) fluid model (Fielding 2005; Fielding and Olmsted 2006; Olmsted et al. 2000; Radulescu and Olmsted 2000; Radulescu et al. 1999) and several other models (Yesilata et al. 2006; Zhou et al. 2008) as well as by experiments (Hu and Lips 2005; Radulescu et al. 2003).

The instability due to the non-monotonic constitutive relationship is qualitatively similar to the thermodynamic instability of the van der Waals gas to which the Maxwell construction applies. Correspondingly, the shear rates of the low and high (slow and fast) bands, $\dot{\gamma}_{\text {low }}$ and $\dot{\gamma}_{h i g h}$, are determined by a simple lever rule, as proved through a theoretical analysis (Cates et al. 1993) and simulations (van den Noort and Briels 2007; Zhou et al. 2008). 
The lever rule is cast in a simple form, $\dot{\gamma}_{a}=w_{\text {low }} \dot{\gamma}_{\text {low }}+w_{\text {high }} \dot{\gamma}_{\text {high }}$, where $w_{\text {low }}$ and $w_{\text {high }}$ are the fractions of low and high shear bands, respectively, and $\dot{\gamma}_{a}$ is the applied (macroscopic) shear rate. The fractions $w_{\text {low }}$ and $w_{\text {high }}$ are determined by the constitutive relationship in a way that the shear stresses in the low and high bands flowing at $\dot{\gamma}_{\text {low }}$ and $\dot{\gamma}_{\text {high }}$ match each other. This matching stress is uniquely determined by the constitutive relationship and independent of the applied shear rate. Very recently, Sato et al. defined phenomenological "order parameters" as linear combination of the shear stress and normal stress difference and formulated a theory of shear banding on the basis of the JS fluid model (Sato et al. 2010). Their theory is analogous to the well established Ginzburg-Landau (GL) type free energy theory (Onuki 2002) and naturally explains the necessity of the non-monotonic constitutive relationship for the shear banding (instability of homogeneous flow) as well as the lever rule. Experimental data seem to be in harmony with these theories (Manneville et al. 2004; Radulescu et al. 2003; Salmon et al. 2003).

Thus, for the wormlike micelles and polymer solutions/blends, the shear banding mechanisms appear to be understood to a considerable depth. However, several uncertain points remain. For example, it is not clear if the shear bands are separated by a sharp boundary (similar to the interface between different phases at equilibrium) and how the molecules orient themselves around the boundary. In fact, for the wormlike micelles, experiments showed that the bands of the shear rate and molecular orientation do not necessarily coincide with each other (Hu and Lips 2005; Lerouge et al. 2004) and the orientation gradually varies in a region much wider than the boundary between the shear rate bands (Lerouge et al. 2004). The other uncertain point is found for the normal stress differences, $N$. The steady state shear stress $\sigma_{\mathrm{s}}(=$ matching stress explained above) is the same in the high and low bands but $N$ may vary according to the gradual change of the orientation. The change of $N$ is compensated by a change of the local pressure (Archer et al. 1995; Olmsted 2008). This fact in turn indicates that the rheological understanding of the shear banding is to be made for both $\sigma_{\mathrm{s}}$ and $N$.

Now, we turn our attention to entangled flexible polymers, the material focused in this study. The shear banding not associated with the flow-induced phase separation (flow-induced concentration gradient) has been reported for entangled polymers (Boukany and Wang 2009b; Ravindranath et al. 2008; Tapadia and Wang 2006). The non-monotonic constitutive relationship resulting in the flow instability could be the origin of the shear banding for entangled polymers, as similar to the situation for the wormlike micelles. In fact, rheological properties have been extensively studied within the context of the tube model (Doi and 
Edwards 1989; McLeish 2002), and the constitutive instability due to disentanglement has been reported (Cates et al. 1993; Doi and Edwards 1989; McLeish and Ball 1986). However, Wang (Wang 2008) proposed the other possible molecular scenario that attributes the shear banding to inhomogeneous structural failure (yielding or rupture) of entanglement network. In this scenario, the entangled polymer is considered to behave as a viscoelastic solid before they relax, and the shear banding and the flow instability are mainly related to the intrinsic inhomogeneity of entanglement networks.

Thus, further work is desired for the shear banding in entangled polymers, and we made a numerical study of the conformational heterogeneity under the shear banding. A molecular model was needed for this purpose. The Rolie-Poly (Rouse Linear Entangled POLYmers) model (Likhtman and Graham 2003), a single-mode version of a full theory based on the tube picture (Graham et al. 2003), is simple/easy to analyze but incorporates all dynamic modes considered in the full theory, reptation, chain stretch, contour length fluctuation, and thermal/convective constraint releases. Thus, we utilized the so-called diffusive Rolie-Poly model incorporating a stress diffusion term (Adams et al. 2008; Adams and Olmsted 2009) to analyze the velocity field/chain conformation in a planar Couette flow. Our focus was placed on a relationship between the conformational heterogeneity (or conformational banding) and the shear rate banding, a subject never investigated so far to our best knowledge. The analysis revealed that the Rolie-Poly model exhibits the shear banding because of its constitutive instability and a boundary between the conformational bands is much broader than that between the shear rate bands. This paper presents details of this finding and discusses a mechanism that determines the boundary widths of the two types of bands.

This paper is organized as follows. At first, the governing equations and the quantities to be examined are summarized. Then, we examine the shear-banding structures and analyze the molecular stretch and orientation, placing our focus on a difference of the boundary widths for the orientational and shear rate bands. Furthermore, we examine effects of the applied shear rate, diffusion constant, and relaxation time on the boundary widths to discuss the difference of the broadness of those bands. We also analyze changes of the first normal stress difference across the streamlines. In addition, we compare the shear banding features due to the constitutive instability (our results) and the structural yielding to make some comments for possible banding mechanisms. Finally, we summarize the results of this paper and refer to future work. 


\section{THEORETICAL}

\section{Basic equations}

In this work, we utilize the Rolie-Poly model to examine the rheological properties and chain conformation under steady shear banding. We consider two-dimensional planar flow in $(x, y)$ coordinates with $x$ and $y$ being the velocity and velocity gradient directions, respectively. This treatment is sufficient to capture the essence of the properties/conformation under shear banding. We assume that the polymeric fluid is incompressible and isothermal. Then, in the steady state, the following equations are satisfied.

$\nabla \cdot \mathbf{u}=0$

$-\nabla p+\nabla \cdot \dot{\mathbf{o}}_{t}=0$

Here, $\mathbf{u}$ is the velocity field, $p$ is the pressure field, and $\mathbf{o}_{t}$ is the stress tensor. The stress tensor in the steady state, $\boldsymbol{o}_{t}$, is determined from the two-dimensional Rolie-Poly model as

$\dot{\mathbf{o}}_{t}=\dot{\mathbf{o}}_{s}+\mathbf{\mathbf { o }}_{p}$,

$\mathbf{o}_{s}=\eta_{s}(\nabla \mathbf{u})^{T}+\nabla \mathbf{u}_{-}$,

$\dot{\mathbf{o}}_{p}=\frac{\eta_{p}}{\tau_{d}}(\mathbf{C}-\mathbf{I})$ with $\mathbf{I}=$ unit tensor,

$\mathbf{u} \cdot \nabla \mathbf{C}=(\nabla \mathbf{u})^{T} \cdot \mathbf{C}+\mathbf{C} \cdot \nabla \mathbf{u}-\frac{1}{\tau_{d}}(\mathbf{C}-\mathbf{I})$

$$
-\frac{2(1-\sqrt{2 / \operatorname{tr} \mathbf{C}})}{\tau_{R}}\left[\mathbf{C}+\beta_{C C R}\left(\frac{\operatorname{tr} \mathbf{C}}{2}\right)^{\delta}(\mathbf{C}-\mathbf{I})\right]+D \nabla^{2} \mathbf{C} .
$$

Here, the subscripts $s$ and $p$, respectively, stand for solvent-like and polymeric components. (Definition of these components is explained later in more detail.) $\eta_{p}$ and $\eta_{s}$ represent the viscosities of these components, while $\tau_{d}$ and $\tau_{R}$ respectively indicate the reptation and Rouse times of the polymeric component. $\beta_{C C R}$ is a parameter representing the magnitude of convective constraint release (CCR), $\delta$ is a parameter specifying the exponent for the relaxation due to the CCR, and $D$ is the diffusion constant. It is convenient to introduce the diffusion length $l_{i}$ as $l_{i}=\sqrt{D / \tau_{d}}$. The remaining part of the paper mostly utilizes $l_{i}$ rather $D$. Here, we should emphasize that the dynamic equation for the concentration field is not involved in our analysis because the main focus of this study is placed on the banded structures due to the constitutive instability, not due to the flow-induced phase separation. As judged from experimental results (Boukany et al. 2008), the concentration fluctuation may play just a 
minor role for some cases of highly concentrated systems (but of course not for all cases).

The quantity $\mathbf{C}$ appearing in Eqs. (5) and (6), that determines the stress of the polymeric component, is the polymer conformation tensor. Its $\alpha \beta$ component in the Cartesian coordinate, with $x$ and $y$ being chosen as the velocity and velocity gradient directions, is defined by

$$
C_{\alpha \beta} \equiv 2\left\langle v_{\alpha} v_{\beta}\right\rangle_{1 \mathrm{st}}, \quad \text { (for two-dimensional problem). }
$$

Here, $\boldsymbol{v}$ is the tangential vector of the chain $(\boldsymbol{v}=\partial \mathbf{R} / \partial s$ with $\mathbf{R}=$ position of the chain segment having the curvilinear coordinate $s$ ), the angular brackets denote an ensemble average, and the subscript "1st" stands for the amplitude of the slowest relaxation mode obtained as the lowest Fourier component of $2\left\langle v_{\alpha}(s) v_{\beta}(s)\right\rangle$ with respect to the Rouse eigenfunctions (Likhtman and Graham 2003). Note that $C_{\alpha \beta}$ given by Eq. (7) is independent of $s$ and that the orientational anisotropy and stretch ratio of the polymer chain are specified by $C_{x y}$ and $\sqrt{\operatorname{tr} \mathbf{C} / 2}=\left\{\left(C_{x x}+C_{y y}\right) / 2\right\}^{1 / 2}$, respectively. (Note also that $C_{\alpha \beta}$ is twice of the components of the usually adopted conformation tensor, $\langle\mathbf{v v}\rangle_{1 s t}$.)

The steady state profiles are obtained by solving Eqs. (1)-(6) numerically with a pseudo-dynamics method. In this method, we regard the fields $\mathbf{u}, \mathbf{C}$, and/or $p$ as dynamic variables and solve the time $(t)$-dependent diffusive Rolie-Poly equation.

$$
\begin{aligned}
\frac{\partial \mathbf{C}}{\partial t}+\mathbf{u} \cdot \nabla \mathbf{C}= & \nabla \mathbf{u}^{T} \cdot \mathbf{C}+\mathbf{C} \cdot \nabla \mathbf{u}-\frac{1}{\tau_{d}}(\mathbf{C}-\mathbf{I}) \\
& -\frac{2(1-\sqrt{2 / \operatorname{tr} \mathbf{C}})}{\tau_{R}}\left(\mathbf{C}+\beta_{C C R}\left(\frac{\operatorname{tr} \mathbf{C}}{2}\right)^{\delta}(\mathbf{C}-\mathbf{I})\right)+D \nabla^{2} \mathbf{C} .
\end{aligned}
$$

At each time, the velocity and pressure fields, $\mathbf{u}$ and $p$, are calculated to satisfy Eqs. (1)-(6). The steady state profiles are obtained in the limit of $t \rightarrow \infty$. The pseudo-dynamic method successfully gave stable and physically reasonable steady state profiles. (For some cases, the method gave just a globally meta-stable profile if two or more locally stable profiles exist. However, this did not give a serious problem for the results presented in this paper.)

Here, the solvent-like, Newtonian component considered in Eq. (4) is rigorously defined. This component is not exclusively defined as a real, low molecular weight solvent. Instead, the viscous response of this component includes a contribution from polymer chains not explicitly treated in the Rolie-Poly model: This single-mode model focuses on the slowest relaxation/motional mode and does not explicitly incorporate contributions from faster modes. 
The viscous response of the solvent-like component expresses the response of the real solvent (if any) as well as the response from such fast modes that should have relaxed in the time scale treated by the single-mode model. Consequently, the polymeric component considered in Eqs. (3)-(6) is defined for the slowest mode of the polymer chain. For convenience of representing a relative contribution of the solvent-like component to the viscosity of the system, we introduce a new parameter,

$$
\phi_{s}=\frac{\eta_{s}}{\eta_{s}+\eta_{p}}
$$

This parameter serves as a parameter representing the entanglement density. (For linear polymers, $\phi_{\mathrm{s}}$ decreases with increasing density and then saturates in the highly entangled regime.)

\section{Calculation method}

For numerically solving Eqs. (1)-(6), we assumed the system to be translationally symmetric in the velocity $(x)$ direction and introduced a one-dimensional mesh in the velocity gradient $(y)$ direction. All fields are expressed as functions of $y$, such as $\mathbf{u}(y), p(y)$, and $\mathbf{C}(y)$. In the pseudo-dynamic calculation explained earlier, the fields were treated to be also dependent on time $t$. The system in the $y$ direction, with the size $L_{y}=1$, was discretized into $N_{\text {elem }}$ (= 800-8000) mesh elements, where the $N_{\text {elem }}$ value was chosen in accordance to the $D$ (or $l_{i}$ ) value so that the diffusion behavior was accurately/properly described.

At the walls forcing the system to flow, a non-slip boundary condition was applied. Specifically, the upper wall was moving at a constant velocity $V_{\text {wall }}$ while the bottom wall was stationary, and the fluid velocities at the walls agreed with these velocities, i.e., $\mathbf{u}\left(L_{y}\right)=\left[V_{\text {wall }}, 0\right]$ and $\mathbf{u}(0)=[0,0]$. We also imposed the Neumann boundary condition, $\partial \mathbf{C} / \partial y=0$ at $y=0$ and $y=L_{y}$ (Adams and Olmsted 2009; Fielding 2005; Olmsted et al. 2000), to prevent the stress flux into the walls. In addition, the pressure at the bottom wall was set to zero, $p(0)=0$.

To obtain the steady state velocity/conformation profiles, the pseudo-dynamic simulation explained earlier was continued for sufficiently long time for each flow condition and each set of material parameters. Convergence of the simulation was monitored with the $L_{2}$-norms of dynamic variables $\mathbf{u}, p$ and $\boldsymbol{o}_{p}$ divided by number of nodes $\left(N_{\text {node }}\right)$. Each simulation run was stopped when the norms became less than a certain tolerance value $\left(10^{-12}\right)$, and the fields $\mathbf{u}, p$ and $\boldsymbol{o}_{p}$ at that time were stored as the steady state fields satisfying Eqs. 
(1)-(6). Further detail of the simulation/calculation is summarized in Appendix.

\section{Parameter values}

The original tube theory (Doi and Edwards 1989) gives the decrease and increase of the shear stress in ranges of the shear rate $\tau_{d}^{-1}<\dot{\gamma}<\tau_{R}^{-1}$ and $\tau_{R}^{-1}<\dot{\gamma}$, respectively, with the increase being due to the fast Rouse modes. Paying attention to this feature, we made the simulation mostly for a set of parameters, $\tau_{d}=100, \tau_{R}=1$ (cf. time scale is non-dimensionalized with $\tau_{R}$ in this study), and also in the whole range of $15 \leq \tau_{d} / \tau_{R} \leq 500$ for some cases to examine the rheological and conformational behavior deduced from the Rolie-Poly model in the negative slope $\left(d \sigma_{t} / d \dot{\gamma}<0\right)$ regime at $\tau_{d}^{-1}<\dot{\gamma}<\tau_{R}^{-1}$. This negative slope vanishes on an increase of the CCR intensity parameter $\beta_{C C R}$ (Likhtman and Graham 2003 ) but can be preserved for $\beta_{C C R} \rightarrow 0$ and $\phi_{s} \rightarrow 0$ (with $\phi_{\mathrm{s}}$ being defined by Eq.(9): (Adams et al. 2008; Adams and Olmsted 2009). Thus, in our simulation, we utilized $\beta_{C C R}=0$ and a sufficiently small $\phi_{s}$ value $\left(=10^{-4}\right)$ to reproduce the shear banding in the negative slope regime. Here, we should notice that the value of the CCR intensity parameter $\left(\beta_{C C R}=0\right)$ in our calculation underestimates the CCR effect. Although very small value of $\beta_{C C R}$ is employed by Likhtman and Graham (Likhtman and Graham 2003) for several high shear rate cases, it is not quantitatively accurate in whole shear rate regime. However, our purpose in this work is to investigate shear-banded structures rather qualitatively, thus we simply employ $\beta_{C C R}=0$. As we discuss later, the main results are expected not to be qualitatively affected by the parameter set. (That is, $\beta_{C C R}=0$ can be reasonably employed in the followings.)

Here, we compare the parameter values utilized in our simulation with the experimental conditions. Experiments (Boukany and Wang 2009b; Ravindranath et al. 2008) revealed the steady state shear banding for highly entangled polymers in ranges of applied shear rate, $0.27 \leq \dot{\gamma}_{a} \tau_{R} \leq 2.65$ for $Z=40,0.51 \leq \dot{\gamma}_{a} \tau_{R} \leq 2.21$ for $Z=70$, and $1.7 \leq \dot{\gamma}_{a} \tau_{R} \leq 17$ for $Z=156$, and so on, where $Z$ is the number of entanglements per chain. (The $\tau_{R}$ specifying these ranges were evaluated from the reported $Z$ and $\tau_{\mathrm{d}}$ values on the basis of the simplest tube model relationship, $\tau_{\mathrm{R}}=\tau_{\mathrm{d}} / 3 Z$.) Thus, the flow condition in our simulation, $0.05 \leq \dot{\gamma}_{a} \tau_{R} \leq 2$, together with the parameters $\phi_{s}=10^{-4}$ and $15 \leq \tau_{d} / \tau_{R} \leq 500$ (mainly $\tau_{d} / \tau_{R}=100$ ), was comparable with the empirical shear-banding condition. 
The diffusion constant $D$ of entangled monodisperse linear polymers is known to depend on $Z$ and $\tau_{\mathrm{d}}$ as $D \propto Z^{-\alpha} \propto Z / \tau_{\mathrm{d}}$ with $\alpha \cong 2.4\left(\tau_{d} \propto Z^{\alpha+1}\right.$ : (Lodge 1999; Wang 2003). Besides, $D$ depends on various parameters such as the polymer density. Thus, we varied $l_{i}=\sqrt{D / \tau_{d}}$ (or $D$ ) as well as the $\tau_{d} / \tau_{R}$ ratio to explore a wide parameter space. Unfortunately, the experimental data of diffusion constants for shear-banded polymer systems are not available, although some experimental methods can provide information for diffusion constants (Tao et al. 2000; Wheeler and Lodge 1989). Therefore, values of $l_{i}$ examined in this study might be somehow unrealistic. However, it turned out that $l_{i}$ affects the rheological/conformational behavior less significantly compared to the $\tau_{d} / \tau_{R}$ ratio. Therefore, we mainly concentrate on the $\tau_{d} / \tau_{R}$ ratio in our later discussion. 


\section{RESULTS AND DISCUSSION}

\section{Constitutive relationship}

In Fig. 1, the normalized shear stress in the steady state, $\sigma_{\mathrm{t}} \tau_{\mathrm{d}} / \eta_{0}$ with $\eta_{0}$ being the zero-shear viscosity, is plotted against the applied (macroscopic) shear rate normalized by $\tau_{\mathrm{R}}$, $\dot{\gamma}_{a} \tau_{R}$. This simulation result was obtained for the parameters, $\tau_{d} / \tau_{R}=100, \phi_{s}=10^{-4}, D=10^{-6}$, and $N_{\text {elem }}=800$. At low and high $\dot{\gamma}_{a}$, the stress increases with increasing $\dot{\gamma}_{a}$ (plots shown with the circles). At those $\dot{\gamma}_{a}$, the system exhibited stabilized homogeneously flow and the local shear rate coincided with $\dot{\gamma}_{a}$. In contrast, at intermediate $\dot{\gamma}_{a}$ where the constitutive relationship of the Rolie-Poly model led to a decrease of the stress $\left(d \sigma_{t} / d \dot{\gamma}<0\right.$; shown with the dotted curve), the stress is constant and independent of $\dot{\gamma}_{a}$ (plots shown with the squares). At the constant stress (stress plateau) region, we observed two regions with different shear rates, which is consistent with previous study (Fielding 2005). This fact suggests that our simulation reproduced the most stable flow profile of the Rolie-Poly model under the shear-banding condition, which lends support to our simulation utilizing the pseudo-dynamic calculation method. (The system can be trapped at meta-stable state if there are two or more locally stable states, as we explained. The reduction theory (Sato et al. 2010) predicts that the banded state is always the globally stable for $\dot{\gamma}_{\text {low }}<\dot{\gamma}<\dot{\gamma}_{\text {high }}$. Therefore we consider the homogeneous flows obtained for $\dot{\gamma}_{\text {low }}<\dot{\gamma}<\dot{\gamma}_{\text {high }}$ are meta-stable. However, the remaining part of this paper focuses on the conformational behavior in the shear-banded regime, and this problem of the global stability does not disturb our discussion.)

\section{Molecular conformation under steady shear banding}

Following a recent study (Adams et al. 2008), Fig. 2 shows viscoelastic ellipses based on the eigenvectors of the polymer conformation tensor, $\mathbf{C}$ ( $c f$. Eq. (7)), obtained from the simulation for the parameters, $\tau_{d} / \tau_{R}=100, \phi_{s}=10^{-4}, D=10^{-6}$ and $N_{\text {elem }}=800$. These parameters are common for the results shown in Figs. 1 and 2.

As noted in Fig. 2, the molecular conformation represented by the ellipses is uniform in the whole range of $y / L_{y}$ (normalized position in the shear gradient direction) at low and high $\dot{\gamma}_{a} \tau_{R}$ where the system exhibited homogeneous flow (cf. Fig. 1). The chains are more stretched and oriented at larger $\dot{\gamma}_{a} \tau_{R}$, as naturally expected. On the other hand, at intermediate $\dot{\gamma}_{a} \tau_{R}$ where the shear banding occurs, the polymer molecule takes different conformation in 
different bands. In the dominant part of each band away from the band boundary, the conformation is uniform and independent of $\dot{\gamma}_{a} \tau_{R}$.

However, we also note an intermediate conformation (more rigorously, conformation averaged over a local ensemble of chains) in the vicinity of the boundary between the shear rate bands; see the ellipses enclosed in dashed ovals in Fig. 2. Thus, the boundary between the conformational bands (two regions of different molecular conformation) is rather broad. This is not a trivial result, because one may expect a sharp change of the molecular conformation similar to that of the shear rate seen on the banding (or, on the flow-induced phase separation described by the Ginzburg-Landau model).

For further investigation of this conformational banding, we focus on the molecular stretch ratio $\Lambda$ and the orientational angle $\Theta$, the former being defined as a ratio of the long axis length of the deformed ellipse under flow to the axis length of the undeformed ellipse at rest, and the latter, as the angle between the long axis of the deformed ellipse and the $x$-axis. (These $\Lambda$ and $\Theta$ are again the averages over the local ensemble of chains.) In Fig. 3, the local shear rate, the stretch ratio $\Lambda$, and the orientation angle $\Theta$ are plotted against the normalized position $y / l_{i}$ in the shear gradient direction. The applied shear rate was $\dot{\gamma}_{a} \tau_{R}=1$, and all other parameters, including $l_{i}=\sqrt{D / \tau_{d}}=10^{-2}$, were the same as those utilized in Figs. 1 and 2 . The whole range spans from $y / l_{i}=0$ to $y / l_{i}=100$, and the plots in Fig. 3 magnify the changes in the vicinity of the boundary between the shear rate bands (for $75 \leq y / l_{i} \leq 85$ ). Clearly, the changes of $\Lambda$ and $\Theta$ with the position are much more gradual compared to that of the local shear rate.

For quantitative comparison of the broadness of the conformational and shear rate banding, we evaluated the width of the band boundary. For the shear rate bands, we fitted the profile shown in Fig. 3(a) with a hyperbolic tangent profile, $\dot{\gamma}(y)=\overline{\dot{\gamma}}+(\Delta \dot{\gamma} / 2) \tanh \left((y-\bar{y}) / l_{0}\right)$ where $\overline{\dot{\gamma}}=\left(\dot{\gamma}_{\text {low }}+\dot{\gamma}_{\text {high }}\right) / 2, \Delta \dot{\gamma}=\left(\dot{\gamma}_{\text {high }}-\dot{\gamma}_{\text {low }}\right), \bar{y}$ represents the location of the center of the boundary, and $l_{0}$ is a parameter representing the broadness of the profile. (This functional form was theoretically suggested in the vicinity of critical point; (Sato et al. 2010)) The fitting was successfully achieved as shown with the thin curve in Fig. 3(a). Thus, we evaluate the boundary width of the shear rate bands as $l_{s r}=2 l_{0}$. A fraction, $\tanh (1)=0.7616(76.16 \%)$, of the total change of the local shear rate, $\Delta \dot{\gamma}$, is achieved on a change in the position by $\Delta y=l_{s r}$ (from $y=\bar{y}-l_{0}$ to $y=\bar{y}+l_{0}$ ). Thus, our $l_{s r}$ can be also defined as a length scale giving $76.16 \%$ of the total change of the local shear rate. 
To determine the boundary width for the molecular conformation bands, $l_{m c}$, we employ the orientation angle $\Theta$ rather than the molecular stretch $\Lambda$, since the $\Theta$ profile seems to change in broader region as shown in Fig. 3(b). Therefore, we expect the $\Theta$ profile reflects the underlying full relaxation more sensitively. (As aforementioned, the conformation tensor $\mathbf{C}$ has several different relaxation modes and we should choose the slowest mode to analyze the relaxation behavior correctly.) The $\Theta$ profile was asymmetric and could not be fitted with the hyperbolic tangent profile, therefore we defined $l_{m c}$ as a length scale giving $76.16 \%$ of the total change, $\Delta \Theta=\Theta(y=0)-\Theta\left(y=L_{\mathrm{y}}\right)$. This definition is in harmony with that for the local shear rate explained above. For evaluation of $l_{m c}$, we chose the boundary center of the shear rate bands located at $\bar{y}_{\mathrm{sr}}$ as a reference point and split the $\Theta$ profile into two profiles at $y<\bar{y}_{s r}$ and $y>\bar{y}_{s r}$ (in the low and high shear bands, respectively; see Fig. 3(b)). Then, we evaluated $l_{m c}^{\text {low }}$ and $l_{m c}^{\text {high }}$ as length scales achieving $76.16 \%$ of the total changes in the low and high shear bands, $\Delta \Theta^{\text {low }}=\Theta(y=0)-\Theta\left(y=\bar{y}_{\text {sr }}\right)$ and $\Delta \Theta^{\text {high }}=\Theta\left(y=\bar{y}_{\text {sr }}\right)-\Theta\left(y=L_{\mathrm{y}}\right)$, respectively, and obtained the boundary width as $l_{m c}=l_{m c}^{\text {low }}+l_{m c}^{\text {high }}$. (Note that this choice of the reference point is necessary to match the definitions for $l_{s r}$ and $l_{m c}$ and that a change in the position by $l_{m c}$ with respect to this reference point gives $76.16 \%$ of the total change, $\Delta \Theta$.)

Fig. 4 shows the normalized boundary widths, $l_{s r} / l_{i}$ and $l_{m c} / l_{i}$, thus obtained for various parameters, $\tau_{d} / \tau_{R}=100, \phi_{\mathrm{s}}=10^{-4}, N_{\text {elem }}=800-8000$ corresponding to $l_{i}=10^{-2}-10^{-3}$, and $\dot{\gamma}_{a} \tau_{R} \leq 2$. Clearly, $l_{m c}$ is considerably larger than $l_{s r}$, confirming the broadness of the conformational bands compared to the shear rate bands. We also note that the widths are quite insensitive to the applied shear rate, $\dot{\gamma}_{a}$. This is physically reasonable, since the applied shear rate affects only on the position of the boundary (via the lever rule) and the shear rate in each band $\left(\dot{\gamma}_{\text {low }}\right.$ and $\dot{\gamma}_{h i g h}$ ) is independent of $\dot{\gamma}_{a}$. Consequently, the conformation in each band including the boundary is independent of $\dot{\gamma}_{a}$ to give the same boundary width as long as the other parameters are the same.

Fig. 4 further demonstrates that the normalized widths, $l_{s r} / l_{i}$ and $l_{m c} / l_{i}$, are quite insensitive to $l_{i}$. Thus, the un-normalized widths are proportional to $l_{i}\left(l_{s r} \cong 0.8 l_{i}\right.$ and $l_{m c} \cong 4.2 l_{i}$ for the parameters examined). This proportionality holds in a wide range of $l_{i}^{2}\left(10^{-2} \leq l_{i} \leq 10^{-3}\right)$, suggesting that the two boundary widths $\left(l_{s r}\right.$ and $\left.l_{m c}\right)$ are dominantly determined by the diffusion constant $D\left(=l_{i}^{2} \tau_{\mathrm{d}}\right)$ appearing in Eq. (6). This diffusion-dominance is consistent with theoretical predictions (Fielding 2005; Sato et al. 2010; Wilson and Fielding 2006). 
Here, we ask a natural question: How/why do the conformation and shear rate bands have different broadness in their boundaries? The diffusion-dominance explained above provides us with a clue to answer this question. In the limit of slow diffusion $(D \rightarrow 0)$, a polymer molecule should stay at the same position $(y)$ along the velocity gradient direction and always adjust its steady state conformation according to the local shear rate $\dot{\gamma}(y)$. For this case, $l_{m c}$ for the conformation band should coincide with $l_{s r}$ for the shear rate band, the latter being determined by the nonlinear relaxation mechanism incorporated in the Rolie-Poly constitutive model. Thus, the difference between $l_{s r}$ and $l_{m c}$ possibly reflects conformational changes of the polymer molecules that occur during their diffusion. In other words, the difference reflects competition between the molecular diffusion and relaxation.

Fig. 5 schematically illustrates this hypothesis. We first consider a polymer molecule at a position $y=\bar{y}_{s r}+l_{s r} / 2$ with $\bar{y}_{s r}$ being the center position of the boundary between the low and high shear rate bands. This molecule is in the high shear band just out of the boundary region and has a highly oriented/stretched conformation corresponding to $\dot{\gamma}_{h i g h}$ in this band. When this molecule diffuses into the low shear rate band, it cannot immediately adjust its conformation to the less oriented/stretched state corresponding to $\dot{\gamma}_{\text {low }}$. Instead, a characteristic time $\tau_{h \rightarrow l}$ for the conformational relaxation is required for this adjustment. Then, the molecule would exhibit one dimensional diffusion (in the $y$ direction) over an average distance $\cong \sqrt{2 D \tau_{h \rightarrow l}}$ during the conformational adjustment, and this distance should contribute the broadness of the conformational band. Similarly, a molecule located at $y=\bar{y}_{s r}-l_{s r} / 2$ (in the low shear rate band) would diffuse in the high shear band over an average distance, $\cong \sqrt{2 D \tau_{l \rightarrow h}}$ with $\tau_{l \rightarrow h}$ being the relaxation time on an increase of the local shear rate to $\dot{\gamma}_{h i g h}$, before it adjust its conformation in that band. This distance should also contribute to the broadness. Thus, the boundary width $l_{m c}$ of the conformational band is expected to be close to the diffusion distance and expressed as $l_{m c} \cong \sqrt{2 D \tau_{h \rightarrow l}}+\sqrt{2 D \tau_{l \rightarrow h}}+l_{s r}$. The last term in this expression, $l_{s r}$, represents a minor correction for the cases of very rapid relaxation $(\tau \rightarrow 0)$ or very slow diffusion $(D \rightarrow 0)$. For these cases, the polymer molecule immediately adjusts its conformation to the local shear rate and $l_{m c}$ should agree with $l_{s r}$.

Here, a comment needs to be added for the above argument. The conformational relaxation during diffusion is analogous to a chemical reaction during diffusion through an interface between separated phases, the latter process being formulated through a diffusion 
equation incorporating the reaction term. This equation describes motion of the reactant starting from any position in the system thereby giving the reactant concentration profile affected by competition between the diffusion and reaction. The conformational relaxation during diffusion can be similarly formulated. However, in this paper, we examine the boundary width of the conformational band on the basis of the approximate argument focusing on the molecules in the vicinity of the boundary. Thus, we should not expect too much accuracy in the numerical prefactor of $\sqrt{2}$ in the relationship, $l_{m c}=\sqrt{2 D \tau_{h \rightarrow l}}+\sqrt{2 D \tau_{l \rightarrow h}}+l_{s r}$. However, the proportionality between $l_{m c}-l_{s r}$ and $\sqrt{D \tau_{h \rightarrow l}}+\sqrt{D \tau_{l \rightarrow h}}$ is essential (and should be deduced also from the sophisticated analysis based on the diffusion equation).

Here, we attempt to compare the boundary width $l_{m c}$ and the diffusion distance $\sqrt{2 D \tau_{h \rightarrow l}}+\sqrt{2 D \tau_{l \rightarrow h}}$. The conformational relaxation time $\tau$ required for this comparison cannot be analytically expressed as a function of the simulation parameters because of the nonlinear feature of the Rolie-Poly model. Thus, we made simple simulation with the pseudo-dynamic method explained earlier to numerically evaluate $\tau$. In this simulation, we first allowed the system to exhibit the homogeneous steady flow at $\dot{\gamma}_{\text {high }}$ (or at $\dot{\gamma}_{\text {low }}$ ) and then switched the applied shear rate to $\dot{\gamma}_{\text {low }}$ (or to $\dot{\gamma}_{\text {high }}$ ) at a time $t=0$. Then, we followed the transient change of the orientation angle $\Theta(t)$ at $t>0$, and the $\Theta(t)$ was approximately described by a single-exponential retardation function, $\Theta(t)=\Theta(0)+\Delta \Theta\{1-\exp (-t / \lambda)\}$ with $\lambda$ being the retardation time. Thus, we determined the time $t_{c}=\lambda$ required to achieve $e$ (=63.21\%) of the total change $\Delta \Theta=\Theta(\infty)-\Theta(0)$, i.e., $\Theta\left(t_{\mathrm{c}}\right)=\Theta(0)+0.6321 \Delta \Theta$. (Here, we notice that the result of this analysis is not affected by definition of $t_{c}$. For instance, even if $t_{c}$ is taken as $\Theta\left(t_{\mathrm{c}}\right)=\Theta(0)+0.7616 \Delta \Theta$ to make a consistency with the definition of $l_{\mathrm{mc}}$, the essential point is still valid.)

In Fig. 6(a), the boundary width $l_{\mathrm{mc}}$ for the conformational band obtained for various $\tau_{\mathrm{d}} / \tau_{\mathrm{R}}$ ratios $(=15-500)$ and different $D$ values $\left(=10^{-5}\right.$ and $\left.10^{-6}\right)$ is plotted against the diffusion distance $\sqrt{2 D \tau_{h \rightarrow l}}+\sqrt{2 D \tau_{l \rightarrow h}}$ evaluated as above. Clearly, the width is essentially a linear function of the diffusion distance. Furthermore, the width subjected to a minor correction explained earlier, $l_{\mathrm{mc}}-l_{\mathrm{sr}}$, is quite insensitive to $D$ and not only proportional but also close in magnitude to the diffusion distance; see Fig. 6(b). A small difference between the observed proportionality constant, $K=\left(l_{m c}-l_{s r}\right) /\left\{\sqrt{2 D \tau_{h \rightarrow l}}+\sqrt{2 D \tau_{l \rightarrow h}}\right\} \cong 2.3$, and that expected from our earlier argument, $K=1$, is not important because of the approximate nature of the 
argument. These results lend support to our hypothesis that the $l_{m c}$ is affected by the competition between the molecular diffusion and relaxation thereby being larger than $l_{s r}$ and the difference between $l_{m c}$ and $l_{s r}$ vanishes in the limit of fast relaxation/slow diffusion.

In relation to the above results, it is also informative to compare two conformational relaxation times, $\tau_{l \rightarrow h}$ and $\tau_{h \rightarrow l}$. For example, $\tau_{l \rightarrow h}=4.0 \times 10^{-3} \tau_{\mathrm{d}}$ and $\tau_{h \rightarrow l}=2.5 \times 10^{0} \tau_{\mathrm{d}}$ $>\tau_{l \rightarrow h}$ for the case of $\tau_{d} / \tau_{R}=100$ and $\phi_{\mathrm{s}}=10^{-4}$. This relationship, $\tau_{l \rightarrow h}<\tau_{h \rightarrow l}$, was found for all sets of parameters examined. In fact, the corresponding difference of the molecular relaxation times on the step-up and step-down of the shear rate has been observed experimentally (Oberhauser et al. 1998).

The difference between $\tau_{l \rightarrow h}$ and $\tau_{h \rightarrow l}$ is a characteristic feature of the diffusive Rolie-Poly equation, Eq. (8). When the conformation tensor $\mathbf{C}$ is the same in the whole space (no conformational banding) and the flow is uniform (no shear rate banding), this equation with $\beta_{\mathrm{CCR}}=0$ (as adopted in this study) is rewritten as

$$
\frac{d \mathbf{C}}{d t}=(\nabla \mathbf{u})^{T} \cdot \mathbf{C}+\mathbf{C} \cdot \nabla \mathbf{u}-\frac{1}{\tau_{d}}(\mathbf{C}-\mathbf{I})-\frac{2(1-\sqrt{2 / \operatorname{tr} \mathbf{C}})}{\tau_{R}} \mathbf{C} .
$$

The last term in the right hand side of Eq. (10) is nonlinear with respect to $\mathbf{C}$ while the other terms are linear to and/or independent of $\mathbf{C}$. Mathematically, the difference between $\tau_{l \rightarrow h}$ and $\tau_{h \rightarrow l}$ deduced from the Rolie-Poly model under homogeneous flow emerges through the nonlinear term.

For further examining how this difference emerges, we decompose $\mathbf{C}(t)$ as $\mathbf{C}(t)=\mathbf{C}_{0}+\delta \mathbf{C}(t)$, where $\mathbf{C}_{0}$ and $\delta \mathbf{C}(t)$ are the time-independent reference part and a small time-dependent perturbation part, respectively. Utilizing this decomposed form of $\mathbf{C}$ in Eq. (10) and retaining only linear terms with respect to $\delta \mathbf{C}(t)$, we find a linearized equation for $\delta \mathbf{C}(t)$ :

$$
\frac{d(\delta \mathbf{C})}{d t}=(\nabla \mathbf{u})^{T} \cdot \delta \mathbf{C}+\delta \mathbf{C} \cdot \nabla \mathbf{u}-\left[\frac{1}{\tau_{d}}+\frac{2\left(1-\sqrt{2 / \operatorname{tr} \mathbf{C}_{0}}\right)}{\tau_{R}}\right] \delta \mathbf{C}-\frac{\sqrt{2}}{\tau_{R}\left(\operatorname{tr} \mathbf{C}_{0}\right)^{3 / 2}} \mathbf{C}_{0} \operatorname{tr} \delta \mathbf{C}
$$

Since $\delta \mathbf{C}$ is a $2 \times 2$ symmetric tensor, it has three independent components. Thus, we can decompose the tensor equation (Eq. (11)) into three linear equations for the components and, in principle, calculate the relaxation times $\tau$ as the reciprocal of the real parts of the eigenvalues associating to those equations. The last term in Eq. (11) becomes negligible under fast shear $\left(\mathbf{C}_{0} /\left(\operatorname{tr} \mathbf{C}_{0}\right)^{3 / 2} \rightarrow 0\right)$. Considering this feature, we neglected the off-diagonal components of 
$\delta \mathbf{C}$ to approximately analyze a relationship between $\tau$ and the shear rate and obtained a simple, analytic form of the eigenvalues. The corresponding longest relaxation time $\tau$ is given by

$$
\frac{1}{\tau} \approx \frac{1}{\tau_{d}}+\frac{2\left(1-\sqrt{2 / \operatorname{tr} \mathbf{C}_{0}}\right)}{\tau_{R}} .
$$

Eq. (12) suggests a decrease of $\tau$ with increasing $\operatorname{tr} \mathbf{C}_{0}$. Consequently, $\tau$ deduced from the Rolie-Poly model decreases when the polymer molecule is subjected to fast homogeneous shear flow thereby being deformed largely. This feature clearly leads to a relationship $\tau_{l \rightarrow h}<\tau_{h \rightarrow l}$ (faster conformational relaxation on step-up of the shear rate than on step-down) observed in our simulation.

Although here we performed analysis for the diffusive Rolie-Poly model, we expect that we have qualitatively similar results for other constitutive models (as long as the constitutive relation is non-monotonic for shear stress and the relaxation is nonlinear). It is fair to mention that the ratio $l_{m c} / l_{s r}$ depends on details of the model and the value of $l_{m c} / l_{s r}$ obtained in this work may differ from experiments. Nevertheless, we consider our results are qualitatively valid, since our simulations or analysis are based on simple and reasonable physical mechanisms which are fairly common for other constitutive models of entangled polymers.

\section{First normal stress difference $\left(\mathbf{N}_{1}\right)$}

In the Rolie-Poly model, the steady state first normal stress difference $\mathrm{N}_{1}$ increases monotonically with increasing shear rate, as different from the behavior of the shear stress (Likhtman and Graham 2003). This monotonic behavior of $\mathrm{N}_{1}$ is noted experimentally for shear banding systems (Tapadia and Wang 2004). Thus, there appears to be no constitutive instabilities originated from the normal stress difference.

Since $\mathrm{N}_{1}$ is exclusively determined by the conformation tensor $\mathbf{C}$ ( $c f$. Eq. (5)), the broad boundary of the conformational bands discussed in the previous sections naturally results in a gradual change of $\mathrm{N}_{1}$ in the velocity gradient direction. As an example, Fig. 7 shows the profile of $\mathrm{N}_{1}$ in this direction obtained from our simulation for $\tau_{d} / \tau_{R}=100, \phi_{s}=10^{-4}, D=10^{-6}, \dot{\gamma}_{a} \tau_{R}=1$ and $N_{\text {elem }}=800$. The gradual change of $N_{1}$ is similar to that noted for the molecular stretch ratio $\Lambda$ (Fig. 3b) obtained for the same set of parameters. This change of $N_{1}$ is compensated by a change of the local pressure.

No literature data can be found for the $\mathrm{N}_{1}$ profile under shear banding. Thus, we here attempt to compare our result (Fig. 7) with a theoretical prediction based on the diffusive 
Johnson-Segalman (JS) model. This model has a monotonic constitutive relationship for $\mathrm{N}_{1}$ and thus predicts different $\mathrm{N}_{1}$ in the high shear and low shear bands (Yuan 1999), as qualitatively similar to our situation. Nevertheless, the reduction theory (Sato et al. 2010) based on the diffusive JS model predicts that the model has only one boundary width scale common to $\mathrm{N}_{1}$ and shear stress. In this sense, the banding behavior of $\mathrm{N}_{1}$ is different for the Rolie-Poly model (utilized in our simulation) and the JS model. This difference appears to reflect a difference of the molecular relaxation mechanisms in these models. The Rolie-Poly model exhibits nonlinear relaxation as discussed earlier, and its constitutive instability essentially results from this nonlinearity. On the other hand, the JS model exhibits linear relaxation, and its instability is attributed to a slippage effect.

Thus, different models appear to exhibit different banding behavior of $\mathrm{N}_{1}$, which in turn suggests that this behavior may serve as a sensitive monitor for differences of the relaxation mechanisms and constitutive instability in various materials. We consider that the $\mathrm{N}_{1}$ behavior depends on the constitutive model in the similar way as the molecular orientation case. Therefore, the information of $\mathrm{N}_{1}$ in shear-banding systems may help us to investigate the molecular relaxation mechanisms. Our analysis and simulation results imply that the molecular orientation or $\mathrm{N}_{1}$ profiles reflect the molecular relaxation mechanism rather strongly. (Namely, these profiles strongly reflect the information of the underlying molecular level dynamics.) Though, it will be practically difficult to directly observe $\mathrm{N}_{1}$ profiles in experiments, we hope some experiments provide information about the $\mathrm{N}_{1}$ profiles and our results are confirmed. For this issue, a further study is desired.

\section{Comments for the yielding and other possible mechanism(s) of shear banding}

The constitutive instability is widely believed to be the origin of the shear banding phenomena, and our simulation results are consistent with this belief. However, Wang and coworkers proposed that yielding (rupture) of entanglement networks is the origin of the shear banding phenomena in highly entangled polymer systems (Wang et al. 2007). They argued that the intrinsic heterogeneity of the entanglement network (such as a distribution of the network strand size) is essential in the relaxation/shear banding of entangled polymers. The effect of this intrinsic heterogeneity is not incorporated in most of constitutive models including the Rolie-Poly model. (Although some effort has been made for incorporation of this effect, the result was not easy to apply to molecular models (Douglas and Hubbard 1991).)

In the scenario by Wang and coworkers, entangled polymers subjected to rapid flow 
$\left(\&_{a}>1 / \tau_{\mathrm{d}}\right)$ exhibit the shear banding through the yielding mechanism. Once the yielding occurs and a fault plane is formed, this plane exists stably. Furthermore, they reported some experimental results supporting their scenario. For example, they observed the fault planes after imposition of large step shear strains (Ravindranath and Wang 2007). The positions of fault planes appear to be rather randomly distributed, which is consistent with their scenario. In steady shear experiments, they reported that differently banding textures (showing different fault planes) emerged at different runs with the same sample (Ravindranath et al. 2008). This feature is also in harmony with the yielding mechanism.

The entangled polymers behave as unrelaxed rubbers in a time scale where the polymer molecules have not attained the large-scale relaxation. From this point of view, the yielding mechanism should capture some part of reality. Concerning this point, however, we should also make several comments. First of all, some experiments showed that the location of the shear band boundary systematically changes with the applied shear rate (Boukany et al. 2008; Boukany and Wang 2009a, b). The yielding mechanism does not straightforwardly results in this systematic change. Furthermore, several meta-stable flow profiles, different from the most stable band profile, may be observed in an experimental time scale $t_{\text {exp }}$, as suggested from the reduction theory (Sato et al. 2010). Such a meta-stable profile can last not forever but for a considerably long time $>t_{\text {exp }}$, as noted from a similar meta-stability known for the phase transition phenomena. The reduction theory also suggests that for some cases, the first stage of the band formation dynamics strongly depends on small perturbations to the initial state (initial condition), as similar to the situation in the spinodal decomposition described by the time-dependent GL equation (Onuki 2002). In analogy with such well-known phenomena, one could argue that the most-stable shear-banded profile (determined by the constitutive instability) may be difficult to be observed experimentally under some conditions and a meta-stable profile similar to a random ensemble of fault planes may emerge with a different mechanism such as the yielding of the entanglement network.

Of course, this argument is one-sided and we do not rule out a possibility that the yielding is the fundamental banding mechanism for entangled polymers. We should emphasize that the "experimentally observed" shear banding (either stable or meta-stable) could results from several different mechanisms, not only the constitutive instability and yielding discussed so far but also some unspecified mechanism that could have a very microscopic origin. (Note that the macroscopic stress related to the thermal tension of each polymer chain fluctuates significantly with time and varies from point to point, meaning that the constant shear stress 
requirement never works in this molecular level.) Furthermore, there are other factors, such as the curvature effect of the Couette geometry, that may affect the shear banding (Adams and Olmsted 2009; Zhou et al. 2008). Thus, it is strongly desired to characterize the "observed" shear banding for many properties without having a pre-assumption of the underlying mechanism. The normal stress difference may serve as an important property for this purpose, as discussed earlier. Further experimental studies along this line as well as more elaborated theoretical studies connecting the phenomena at molecular and macroscopic levels (including the macroscopic yielding) are strongly desired. 


\section{CONCLUDING REMARKS}

We have utilized the diffusive Rolie-Poly model to simulate the flow behavior and investigate properties related to the boundary between shear bands. This model gave the shear banding due to its constitutive instability. Our simulation showed that the shear rate and conformational bands had quite different widths of their boundaries: The latter was much broader than the former. As a result, the first normal stress difference determined by the polymer conformation exhibited broad banding similar to the conformational banding. Detailed analysis revealed that the difference of the broadness of the shear rate and conformational bands resulted from competition of the molecular diffusion and relaxation mechanism affecting the conformational band. The stability and meta-stability of the shear banding phenomena were also discussed briefly. Although our simulation results depend on the employed constitutive model and parameter sets, we consider our results are qualitatively unchanged for other constitutive models or parameter sets. Therefore, the presented analysis would be meaningful to understand the molecular level relaxation mechanism in shear-banded systems.

\section{Acknowledgements}

This work was partly supported by Grant-in-Aid for Scientific Research on Priority Area "Soft Matter Physics" from the Ministry of Education, Culture, Sports, Science and Technology (grant \#18068009). C. Chung thanks a financial support from G-COE program for the stay at ICR. 


\section{Appendix: Calculation details in simulation}

The finite element method was employed to discretize the governing equations (1)-(6) with stabilizing schemes for viscoelastic fluids such as DEVSS-G (Liu et al. 1998) and SUPG (Brooks and Hughes 1982). We reformulate the continuity Eq. (1) and the momentum Eq. (2) with DEVSS-G scheme (Liu et al. 1998) into the following weak form:

$$
\begin{aligned}
& \langle\phi ; \nabla \cdot \mathbf{u}\rangle=0, \\
& -\langle\nabla \psi ; p \mathbf{I}\rangle+\left\langle\nabla \psi ; \eta\left(\nabla \mathbf{u}+\nabla \mathbf{u}^{T}\right)-\eta \phi_{p}\left(\mathbf{G}^{T}+\mathbf{G}\right)\right\rangle+\left\langle\nabla \psi ; \dot{\mathbf{o}}_{p}\right\rangle=0, \\
& \left\langle\phi ; \mathbf{G}-\nabla \mathbf{u}^{T}\right\rangle=0,
\end{aligned}
$$

where $\phi$ and $\psi$ are linear and quadratic shape functions, respectively, and $\langle;\rangle$ denotes integral along the finite elements. Variables such as $p, \mathbf{G}$ (the velocity gradient tensor), $\mathbf{o}_{p}$ are approximated in terms of linear shape functions, while $\mathbf{u}$ is discretized with quadratic shape function.

We also employed the matrix logarithm (Hulsen et al. 2005) to enhance the numerical stability of calculation. The conformation tensor $\mathbf{C}$ can be diagonalized with the relationship $\mathbf{C}=\mathbf{R} \cdot \mathbf{c} \cdot \mathbf{R}^{T}$, where $\mathbf{R}$ is a matrix composed of the eigenvectors of $\mathbf{C}$ and the diagonal tensor c have the corresponding eigenvalues $c_{\mathrm{i}}$ as its components. We can replace the $\mathbf{C}$-based constitutive model with the logarithm tensor based formulation. Thus, we dealt with the evolution equation of $\mathbf{s}=\log \mathbf{c}=\sum_{i=1}^{2} \log \left(c_{i}\right) \mathbf{n}_{i} \mathbf{n}_{i}=\sum_{i=1}^{2} s_{i} \mathbf{n}_{i} \mathbf{n}_{i}$, with $\mathbf{s}, s_{i}$, and $\mathbf{n}_{i}$ being the logarithm tensor in the principal frame, the eigenvalue of the logarithm tensor, and the principal direction conjugated with the eigenvalues $c_{i}$ of $\mathbf{C}$. The time derivative of $\mathbf{s}$ for the Rolie-Poly model can be written as

$$
\begin{aligned}
\dot{\mathbf{s}} & =\sum_{i=1}^{2}\left(2 G_{i i}-\frac{1}{c_{i}}\left(\frac{1}{\tau_{d}}\left(c_{i}-1\right)\right)-\frac{2\left(1-\sqrt{2 /\left(c_{1}+c_{2}\right)}\right)}{\tau_{R}}\left(c_{i}+\beta_{C C R}\left(\frac{c_{1}+c_{2}}{2}\right)^{\delta}\left(c_{i}-1\right)\right) \frac{1}{c_{i}}\right) \mathbf{n}_{i} \mathbf{n}_{i},\left(\begin{array}{ll}
\mathrm{A} 4 \\
4
\end{array}\right) \\
& +\sum_{i=1}^{2} \sum_{\substack{j=1 \\
i \neq j}}^{2} \frac{s_{i}-s_{j}}{c_{i}-c_{j}}\left(c_{j} G_{i j}+c_{i} G_{j i}\right) \mathbf{n}_{i} \mathbf{n}_{j} .
\end{aligned}
$$

Here, $G_{i j}$ is the components of the velocity gradient tensor in the principal frame. Consequently, the constitutive model with the diffusive term is described by the logarithm tensor $\mathbf{S}$ in the global frame as 
$\frac{\partial \mathbf{S}}{\partial t}+\mathbf{u} \cdot \nabla \mathbf{S}=\dot{\mathbf{S}}+D \nabla^{2} \mathbf{S}$

where $\dot{\mathbf{S}}$ is the tensor transformed from $\dot{\mathbf{S}}$ through the matrix diagonalization; $\dot{\mathbf{S}}=\mathbf{R} \cdot \dot{\mathbf{s}} \cdot \mathbf{R}^{T}$. The discrete form of Eq. (A5) with SUPG scheme (Brooks and Hughes 1982) can be written as $\left\langle\phi+\phi^{s} ; \frac{\mathbf{S}^{n+1}-\mathbf{S}^{n}}{\Delta t}+\mathbf{u}^{n+1} \cdot \nabla \mathbf{S}^{n+1}\right\rangle=\left\langle\phi+\phi^{s} ; \dot{\mathbf{S}}^{n}+D \nabla^{2} \mathbf{S}^{n}\right\rangle$.

Here, $\phi^{s}$ is the element-wise upwinding shape function, $\phi^{s}=\alpha\left(\left|\mathbf{u}_{c} \cdot \mathbf{h}\right|\right) /\left(2 \mathbf{u}_{c} \cdot \mathbf{u}_{c}\right), \mathbf{u}_{c}$ is the velocity vector at center node of an element, and $\mathbf{h}$ is a characteristic size of the element. Following previous literatures (Baaijens 1998; Chung et al. 2008; Kim et al. 2004; Ramirez and Laso 2005), we utilized the streamline upwinding coefficient $\alpha=2$ in Eq. (A6). The superscripts $n$ and $n+1$ appearing in Eq. (A6) denote the present and the next time steps, respectively.

The numerical solution of Eq. (A6) was transformed into the principal frame through a relationship $\mathbf{S}=\mathbf{R}^{T} \cdot \mathbf{S} \cdot \mathbf{R}$ to obtain the conformation tensor $\mathbf{C}\left(=\mathbf{R} \cdot \mathbf{c} \cdot \mathbf{R}^{T}=\mathbf{R} \cdot e^{\mathbf{s}} \cdot \mathbf{R}^{T}\right)$. Finally, the stress tensor for the polymeric component, $\boldsymbol{o}_{p}$, was calculated by Eq. (5). Then, the set of the desired variables, $\mathbf{G}$, $\mathbf{u}$, and $p$, was obtained after solving the coupled Eqs. (A1)-(A3) at every time step. 


\section{References}

Adams JM, Fielding SM, Olmsted PD (2008) The interplay between boundary conditions and flow geometries in shear banding: Hysteresis, band configurations, and surface transitions. J. Non-Newtonian Fluid Mech. 151(1-3):101-118

Adams JM, Olmsted PD (2009) Nonmonotonic models are not necessary to obtain shear banding phenomena in entangled polymer solutions. Phys. Rev. Lett. 102(6):067801

Archer LA, Larson RG, Chen YL (1995) Direct measurements of slip in sheared polymer solutions. J. Fluid Mech. 301133-151

Baaijens FPT (1998) Mixed finite element methods for viscoelastic flow analysis: A review. J. Non-Newtonian Fluid Mech. 79(2-3):361-385

Berret JF, Roux DC, Porte G, Lindner P (1994) Shear-induced isotropic-to-nematic phase transition in equilibrium polymers. Europhys. Lett. 25(7):521-526

Boukany PE, Hu YT, Wang SQ (2008) Observations of wall slip and shear banding in an entangled DNA solution. Macromolecules 41(7):2644-2650

Boukany PE, Wang SQ (2009a) Exploring the transition from wall slip to bulk shearing banding in well-entangled DNA solutions. Soft Matter 5(4):780-789

Boukany PE, Wang SQ (2009b) Shear banding or not in entangled DNA solutions depending on the level of entanglement. J. Rheol. 53(1):73-83

Brooks AN, Hughes TJR (1982) Streamline upwind Petrov-Galerkin formulations for convection dominated flows with particular emphasis on the incompressible Navier-Stokes equations. Comput. Method Appl. Mech. Engrg. 32(1-3):199-259

Cappelaere E, Berret JF, Decruppe JP, Cressely R, Lindner P (1997) Rheology, birefringence, and small-angle neutron scattering in a charged micellar system: Evidence of a shear-induced phase transition. Phys. Rev. E 56(2):1869-1878

Cates ME, Mcleish TCB, Marrucci G (1993) The rheology of entangled polymers at very high shear rates. Europhys. Lett. 21(4):451-456

Chung C, Hulsen MA, Kim JM, Ahn KH, Lee SJ (2008) Numerical study on the effect of viscoelasticity on drop deformation in simple shear and 5:1:5 planar contraction/expansion microchannel. J. Non-Newtonian Fluid Mech. 15580-93

Doi M, Edwards SF (1989) The theory of polymer dynamics. Clarendon Press, Oxford

Douglas JF, Hubbard JB (1991) Semiempirical theory of relaxation: Concentrated polymer solution dynamics. Macromolecules 24(11):3163-3177

Fielding SM (2005) Linear instability of planar shear banded flow. Phys. Rev. Lett. 95(13): 134501

Fielding SM, Olmsted PD (2003a) Early stage kinetics in a unified model of shear-induced demixing and mechanical shear banding instabilities. Phys. Rev. Lett. 90(22):224501

Fielding SM, Olmsted PD (2003b) Kinetics of the shear banding instability in startup flows. Phys. Rev. E 68(3):036313 
Fielding SM, Olmsted PD (2006) Nonlinear dynamics of an interface between shear bands. Phys. Rev. Lett. 96(10):104502

Fischer E, Callaghan PT (2001) Shear banding and the isotropic-to-nematic transition in wormlike micelles. Phys. Rev. E 6401(1):011501

Furukawa A, Onuki A (2005) Spatio-temporal structures in sheared polymer systems. Physica D 205(1-4):195-206

Graham RS, Likhtman AE, McLeish TCB, Milner ST (2003) Microscopic theory of linear, entangled polymer chains under rapid deformation including chain stretch and convective constraint release. J. Rheol. 47(5):1171-1200

$\mathrm{Hu}$ YT, Lips A (2005) Kinetics and mechanism of shear banding in an entangled micellar solution. J. Rheol. 49(5):1001-1027

Hulsen MA, Fattal R, Kupferman R (2005) Flow of viscoelastic fluids past a cylinder at high Weissenberg number: stabilized simulations using matrix logarithms. J. Non-Newtonian Fluid Mech. 127(1):27-39

Jupp L, Yuan XF (2004) Dynamic phase separation of a binary polymer liquid with asymmetric composition under rheometric flow. J. Non-Newtonian Fluid Mech. 124(1-3):93-101

Kim JM, Kim C, Ahn KH, Lee SJ (2004) An efficient iterative solver and high-resolution computations of the Oldroyd-B fluid flow past a confined cylinder. J. Non-Newtonian Fluid Mech. 123(2-3):161-173

Lerouge S, Decruppe JP, Olmsted P (2004) Birefringence banding in a micellar solution or the complexity of heterogeneous flows. Langmuir 20(26):11355-11365

Liberatore MW, Nettesheim F, Wagner NJ, Porcar L (2006) Spatially resolved small-angle neutron scattering in the 1-2 plane: A study of shear-induced phase-separating wormlike micelles. Phys. Rev. E 73(2):020504

Likhtman AE, Graham RS (2003) Simple constitutive equation for linear polymer melts derived from molecular theory: Rolie-Poly equation. J. Non-Newtonian Fluid Mech. 114(1):1-12

Liu AW, Bornside DE, Armstrong RC, Brown RA (1998) Viscoelastic flow of polymer solutions around a periodic, linear array of cylinders: comparisons of predictions for microstructure and flow fields. J. Non-Newtonian Fluid Mech. 77(3):153-190

Lodge TP (1999) Reconciliation of the molecular weight dependence of diffusion and viscosity in entangled polymers. Phys. Rev. Lett. 83(16):3218-3221

Lu CYD, Olmsted PD, Ball RC (2000) Effects of nonlocal stress on the determination of shear banding flow. Phys. Rev. Lett. 84(4):642-645

Manneville S, Salmon JB, Becu L, Colin A, Molino F (2004) Inhomogeneous flows in sheared complex fluids. Rheol. Acta 43(5):408-416

McLeish TCB (2002) Tube theory of entangled polymer dynamics. Adv. Phys. 
51(6):1379-1527

McLeish TCB, Ball RC (1986) A molecular approach to the spurt effect in polymer melt flow. J. Polym. Sci. Pol. Phys. 24(8):1735-1745

Miller E, Rothstein JP (2007) Transient evolution of shear-banding wormlike micellar solutions. J. Non-Newtonian Fluid Mech. 143(1):22-37

Oberhauser JP, Leal LG, Mead DW (1998) The response of entangled polymer solutions to step changes of shear rate: Signatures of segmental stretch? J. Polym. Sci. Pol. Phys. $36(2): 265-280$

Olmsted PD (2008) Perspectives on shear banding in complex fluids. Rheol. Acta 47(3):283-300

Olmsted PD, Radulescu O, Lu CYD (2000) Johnson-Segalman model with a diffusion term in cylindrical Couette flow. J. Rheol. 44(2):257-275

Onuki A (2002) Phase Transition Dynamics. Cambridge University Press, Cambridge

Radulescu O, Olmsted PD (2000) Matched asymptotic solutions for the steady banded flow of the diffusive Johnson-Segalman model in various geometries. J. Non-Newtonian Fluid Mech. 91(2-3):143-164

Radulescu O, Olmsted PD, Decruppe JP, Lerouge S, Berret JF, Porte G (2003) Time scales in shear banding of wormlike micelles. Europhys. Lett. 62(2):230-236

Radulescu O, Olmsted PD, Lu CYD (1999) Shear banding in reaction-diffusion models. Rheol. Acta 38(6):606-613

Ramirez J, Laso M (2005) Size reduction methods for the implicit time-dependent simulation of micro-macro viscoelastic flow problems. J. Non-Newtonian Fluid Mech. 127(1):41-49

Ravindranath S, Wang SQ (2007) What are the origins of stress relaxation behaviors in step shear of entangled polymer solutions? Macromolecules 40(22):8031-8039

Ravindranath S, Wang SQ, Ofechnowicz M, Quirk RP (2008) Banding in simple steady shear of entangled polymer solutions. Macromolecules 41(7):2663-2670

Salmon JB, Colin A, Manneville S, Molino F (2003) Velocity profiles in shear-banding wormlike micelles. Phys. Rev. Lett. 90(22):228303

Sato K, Yuan XF, Kawakatsu T (2010) Why does shear banding behave like first-order phase transition? Deviation of a potention from a mechanical constitutive model. Eur. Phys. J. E 31135-144

Schmitt V, Marques CM, Lequeux F (1995) Shear-induced phase separation of complex fluids: The role of flow-concentration coupling. Phys. Rev. E 52(4):4009-4015

Takenaka M, Nishitsuji S, Taniguchi T, Yamaguchi M, Tada K, Hashimoto T (2006) Computer simulation study on the shear-induced phase separation in semidilute polymer solutions in 3-dimensional space. Polymer 47(22):7846-7852

Tao H, Lodge TP, von Meerwall ED (2000) Diffusivity and viscosity of concentrated 
hydrogenated polybutadiene solutions. Macromolecules 33(5):1747-1758

Tapadia P, Wang SQ (2006) Direct visualization of continuous simple shear in non-newtonian polymeric fluids. Phys. Rev. Lett. 96(1):016001

Tapadia P, Wang SQ (2004) Nonlinear flow behavior of entangled polymer solutions: Yieldlike entanglement-disentanglement transition. Macromolecules 37(24):9083-9095 Tapadia P, Wang SQ (2003) Yieldlike constitutive transition in shear flow of entangled polymeric fluids. Phys. Rev. Lett. 91(19):198301

van den Noort A, Briels WJ (2007) Coarse-grained simulations of elongational viscosities, superposition rheology and shear banding in model core-shell systems. Macromol. Theory Simul. 16(8):742-754

Wang SQ (2003) Chain dynamics in entangled polymers: Diffusion versus rheology and their comparison. J. Polym. Sci. B: Polm. Phys. 41(14):1589-1604

Wang SQ (2008) The tip of lceberg in nonlinear polymer rheology: Entangled liquids are "solids". J. Polym. Sci: Part B: Polym. Phys. 462660-2665

Wang SQ, Ravindranath S, Wang YY, Boukany PY (2007) New theoretical considerations in polymer rheology: Elastic breakdown of chain entanglement network. J. Chem. Phys. 127(6):064903

Wheeler LM, Lodge TP (1989) Tracer diffusion of linear polystyrenes in dilute, semidilute, and concentrated poly(vinyl methyl ether) solutions. Macromolecules 22(8):3399-3408

Wilson HJ, Fielding SM (2006) Linear instability of planar shear banded flow of both diffusive and non-diffusive Johnson-Segalman fluids. J. Non-Newtonian Fluid Mech. 138(2-3):181-196

Yerushalmi J, Katz S, Shinnar R (1970) Stability of steady shear flows of some viscoelastic fluids. Chem. Eng. Sci. 25(12):1891-1902

Yesilata B, Clasen C, McKinley GH (2006) Nonlinear shear and extensional flow dynamics of wormlike surfactant solutions. J. Non-Newtonian Fluid Mech. 133(2-3):73-90

Yuan XF (1999) Dynamics of a mechanical interface in shear-banded flow. Europhys. Lett. 46(4):542-548

Zhou L, Vasquez PA, Cook LP, McKinley GH (2008) Modeling the inhomogeneous response and formation of shear bands in steady and transient flows of entangled liquids. J. Rheol. 52(2):591-623 


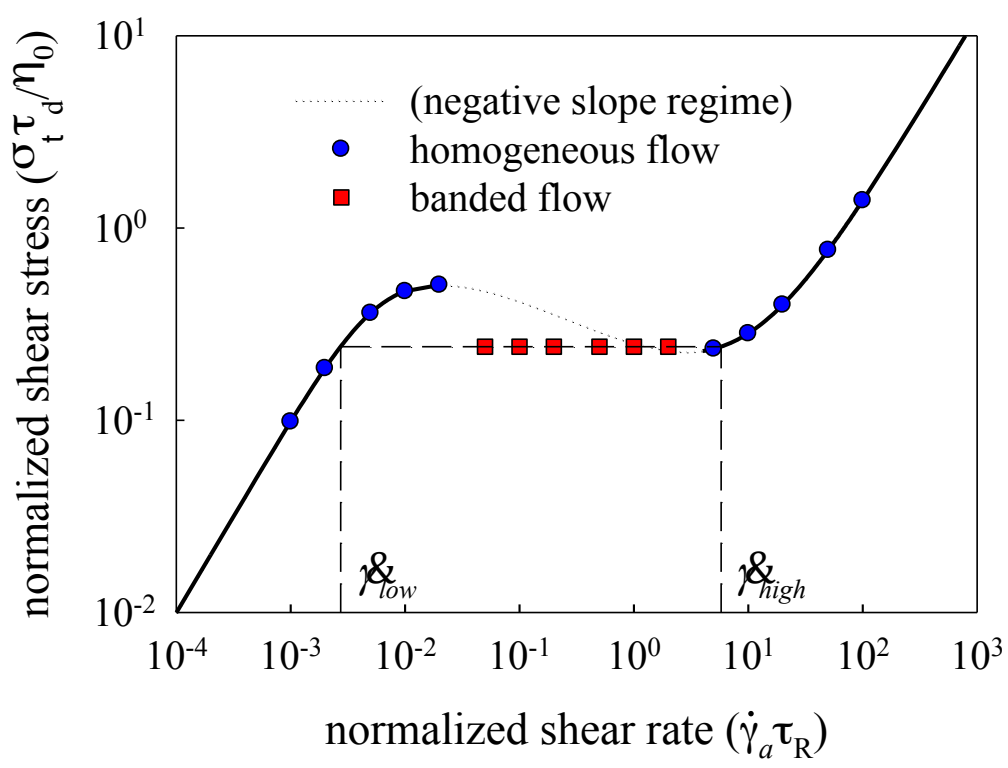

Fig. 1. Constitutive relationship obtained from the flow simulation based on the Rolie-Poly $\operatorname{model}\left(\tau_{d} / \tau_{R}=100, \phi_{s}=10^{-4}, D=10^{-6}\right.$ and $\left.N_{\text {elem }}=800\right)$. 


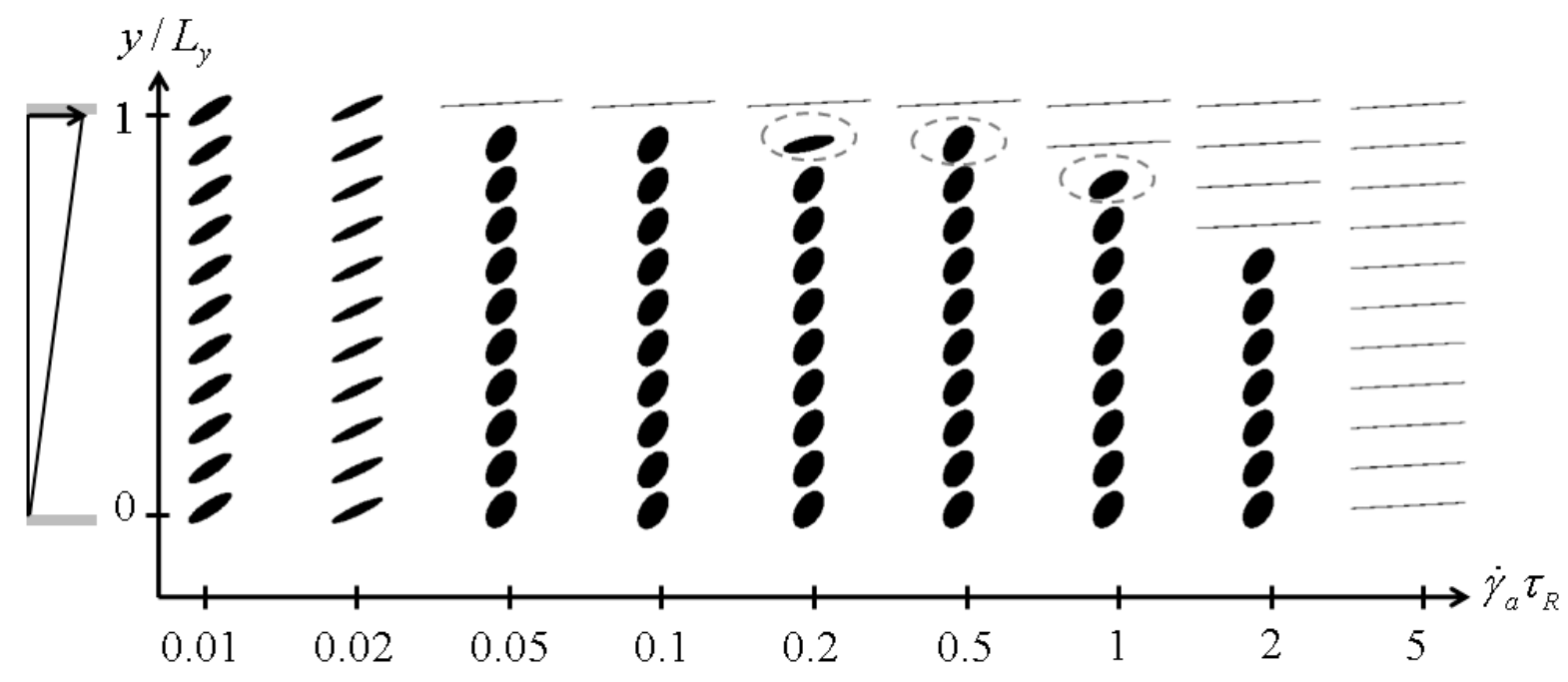

Fig. 2. Molecular conformation depicted as the stress ellipse at various positions under various applied shear rate $\left(\tau_{d} / \tau_{R}=100,=10^{-4}, D=10^{-6}\right.$ and $\left.N_{\text {elem }}=800\right)$. 


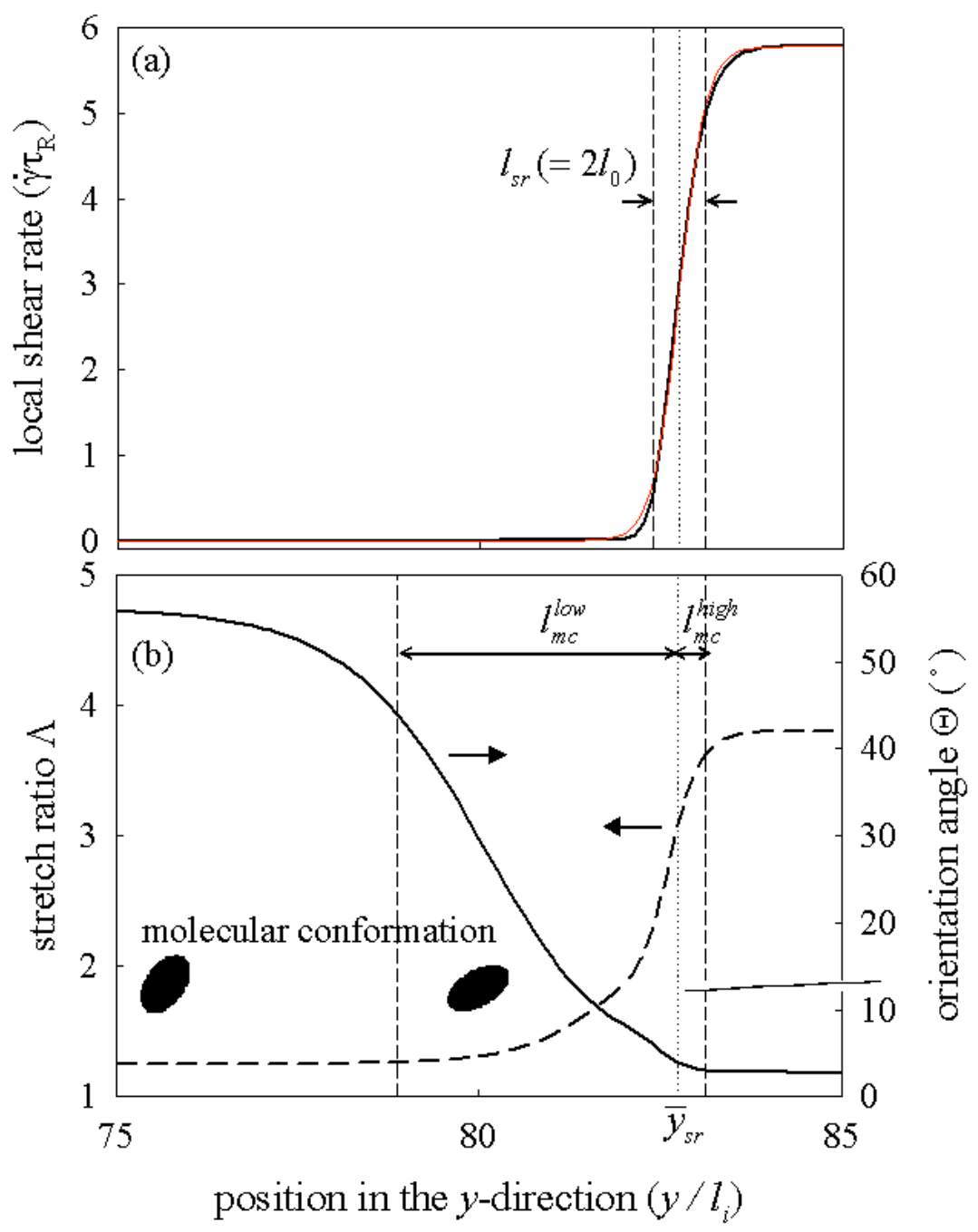

Fig. 3. Profiles of (a) local shear rate and (b) stretch ratio $\Lambda$ and orientation angle $\Theta$ near the boundary between shear rate bands $\left(\tau_{d} / \tau_{R}=100, \phi_{s}=10^{-4}, D=10^{-6}, \dot{\gamma}_{a} \tau_{R}=1\right.$ and $\left.N_{\text {elem }}=800\right)$. The boundary width for the shear rate $\left(l_{s r}\right)$ is estimated by fitting the profile with the hyperbolic tangent function (thin curve). In part $\mathrm{b}$, the characteristic lengths $l_{m c}^{l o w}$ and $l_{m c}^{\text {high }}$, respectively, are defined as length scales achieving $76.16 \%$ of the total change of $\Theta$ in the regimes $y<\bar{y}_{s r}$ and $y \geq \bar{y}_{s r}$, where $\bar{y}_{s r}$ is the boundary center position of the shear rate bands (cf. part a). The boundary width for the conformational band (defined for $\Theta$ ) is given by $l_{m c}=l_{m c}^{\text {low }}+l_{m c}^{\text {high }}$. 


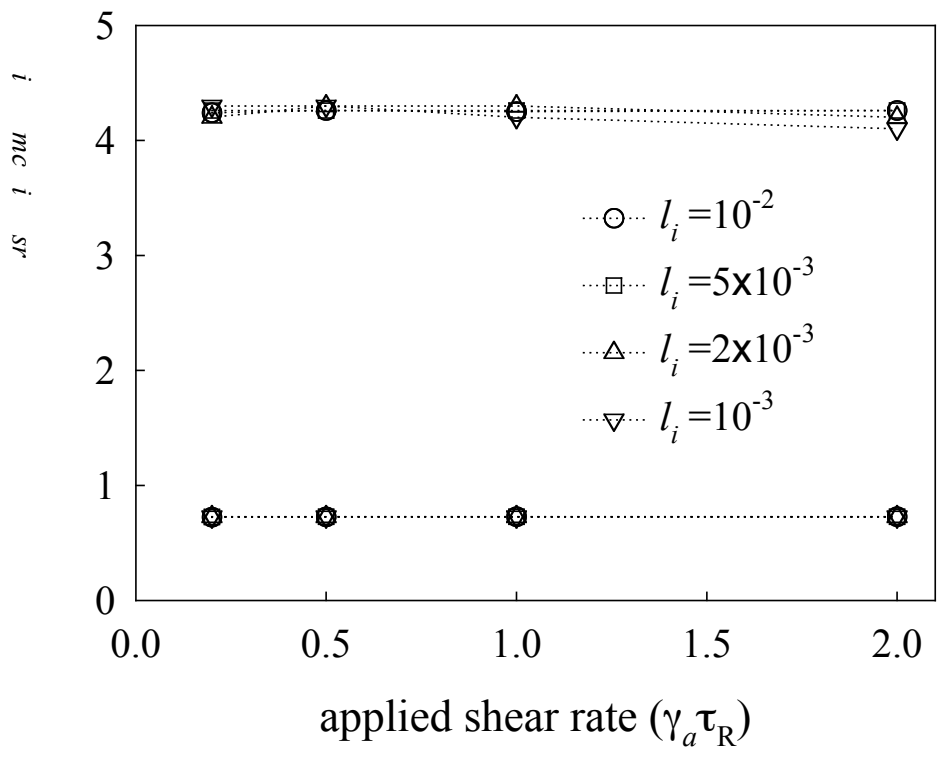

Fig. 4. Effect of the applied shear rate $\left(\dot{\gamma}_{a} \tau_{R}\right)$ and $l_{i}$ on the boundary widths, $l_{s r}$ and $l_{m c}$. The parameter set are $\tau_{d} / \tau_{R}=100, \phi_{s}=10^{-4}$ and $N_{\text {elem }}=800,1600,4000,8000$ for $l_{i}=10^{-2}, 5 \times 10^{-3}$, $2 \times 10^{-3}, 10^{-3}$, respectively (i.e., $D=10^{-6} \sim 10^{-8}$ ). 


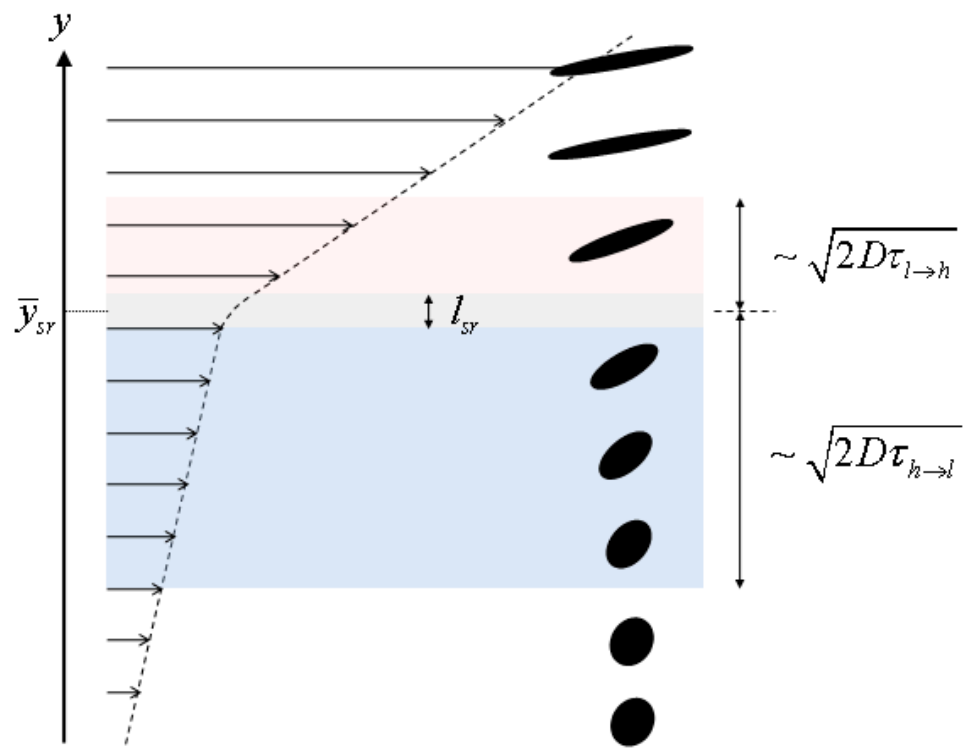

Fig. 5. Schematic diagram showing competition of molecular diffusion and relaxation. $l_{s r}$ and $l_{m c}$ are the boundary widths for the shear rate and conformation bands, respectively. $\tau_{h \rightarrow l}$ and $\tau_{l \rightarrow h}$ are the characteristic relaxation times of molecular conformation on a switch of local shear rate, from $\dot{\gamma}_{\text {high }}$ to $\dot{\gamma}_{\text {low }}$ and from $\dot{\gamma}_{\text {low }}$ to $\dot{\gamma}_{\text {high }}$, respectively. 

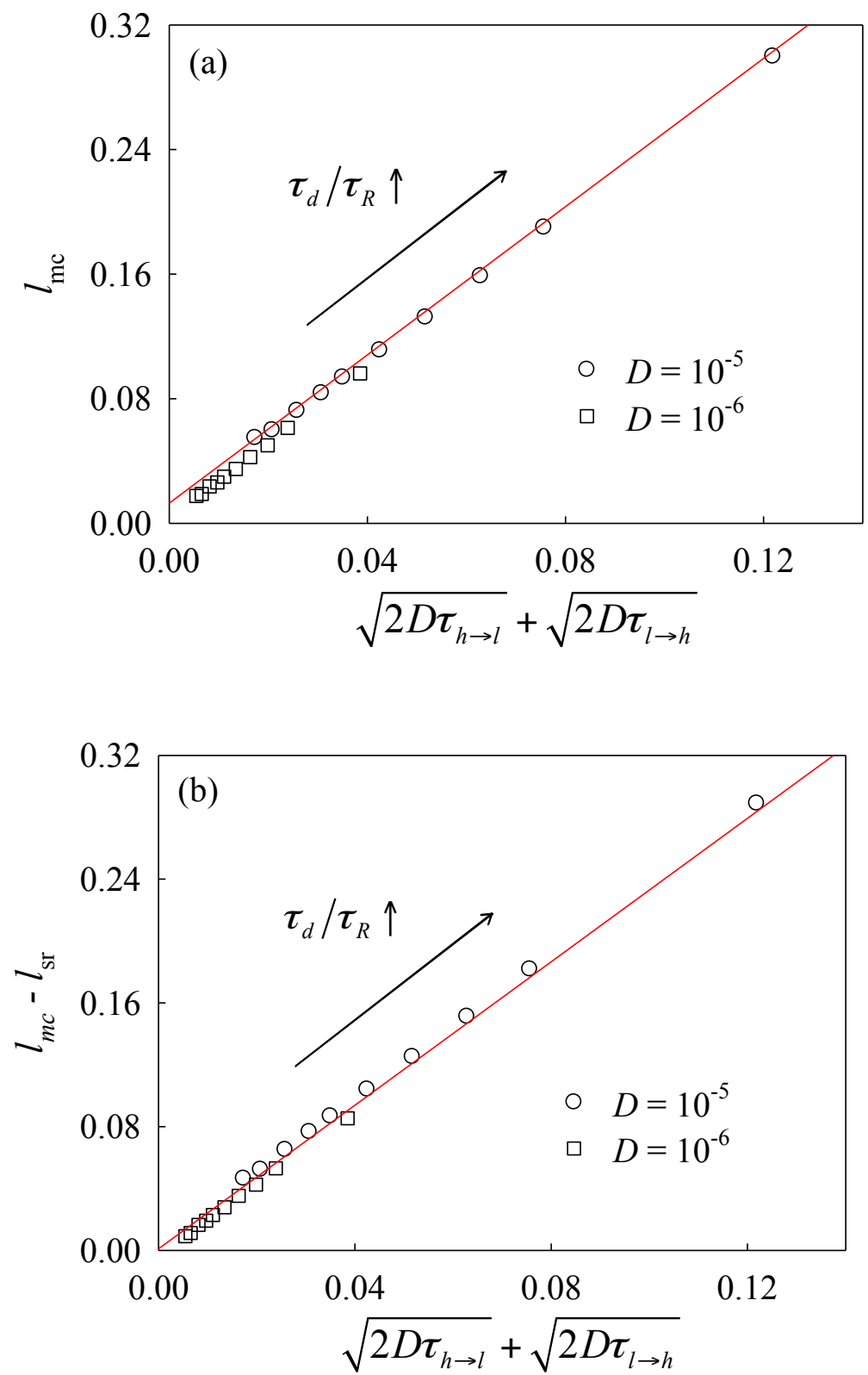

Fig. 6. Dependence of (a) $l_{m c}$ and (b) $l_{m c} l_{s r}$ on the diffusion distance in the time scale of molecular relaxation. The parameter set is $15 \leq \tau_{d} / \tau_{R} \leq 500, \phi_{s}=10^{-4}$ and $N_{\text {elem }}=800$. 


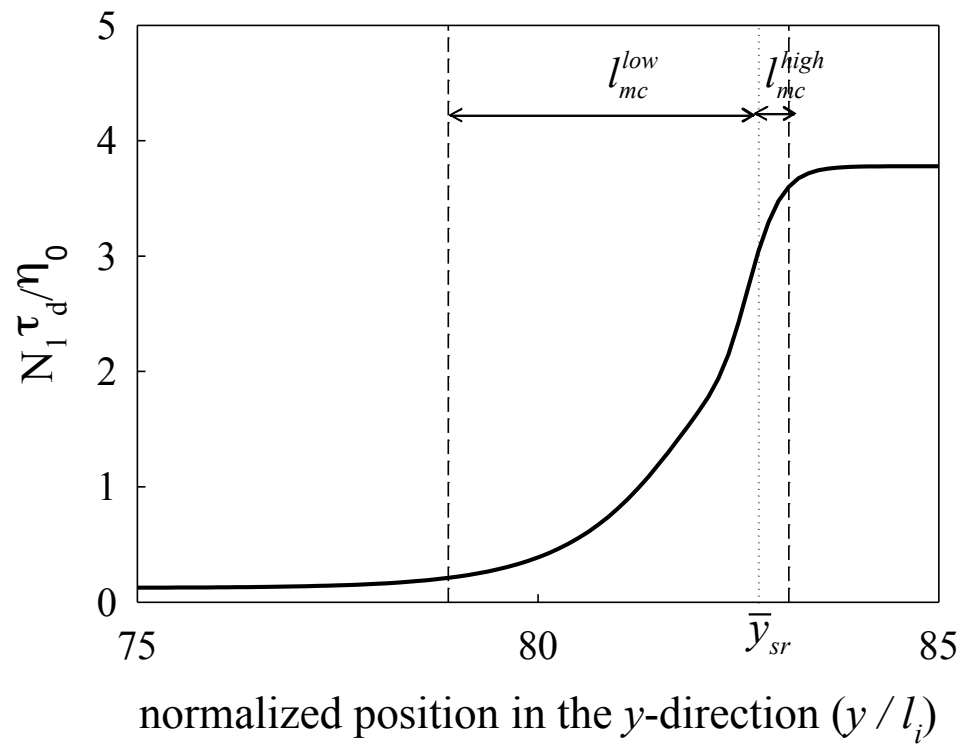

Fig. 7. The profile of $\mathrm{N}_{1}$ across the boundary of the shear rate bands. The parameter set is $\tau_{d} / \tau_{R}=100, \phi_{s}=10^{-4}, D=10^{-6}, \dot{\gamma}_{a} \tau_{R}=1$ and $N_{\text {elem }}=800$. 